\title{
Parallaxes of Brown Dwarfs at USNO
}

Hugh C. Harris, Conard C. Dahn, Frederick J. Vrba, Harry H. Guetter, Blaise Canzian, Arne A. Henden, Stephen E. Levine, Christian B.

Luginbuhl, Alice K. B. Monet, David G. Monet, Jeffery R. Pier, Ronald C. Stone, Richard L. Walker

U. S. Naval Observatory, P.O. Box 1149, Flagstaff, AZ 86002-1149

\begin{abstract}
Trigonometric parallaxes have been measured by Dahn et al. (2002) for 28 cool dwarfs and brown dwarfs, including $17 \mathrm{~L}$ dwarfs and three T dwarfs. Broadband CCD and near-IR photometry $\left(V R I z^{*} J H K\right)$ have been obtained for these objects and for 24 additional late-type dwarfs. These data have been supplemented with astrometry and photometry from the literature, including parallaxes for the brighter companions of ten $\mathrm{L}$ and two $\mathrm{T}$ dwarfs. The absolute magnitudes and colors are reviewed here. The $I-J$ color and the spectral type are both good predictors of absolute magnitude for late-M and L dwarfs. $M_{J}$ becomes monotonically fainter with $I-J$ color and with spectral type through late$\mathrm{L}$ dwarfs, then brightens for early-T dwarfs. In contrast, the $J-K$ color correlates poorly with absolute magnitude for L dwarfs. Using several other parameters from the literature (Li detection, $\mathrm{H} \alpha$ emission strength, projected rotation velocity, and tangential velocity), we fail to uncover any measurable parameter that correlates with the anomalous $J-K$ color.
\end{abstract}

\section{Introduction}

Parallaxes of late-M, L, and $\mathrm{T}$ dwarfs have been measured from images taken over the past several years with the $1.55 \mathrm{~m}$ Strand astrometric telescope at the U.S. Naval Observatory. The present results have been published recently by Dahn et al. (2002). The parallaxes are necessary for many purposes, including comparison with evolutionary models, analyzing the kinematics, determining ages, determining temperatures, and identifying outlying objects (perhaps binaries or young objects). When faint companions to bright stars with Hipparcos parallaxes are included, there are now nearly 30 field $L$ dwarfs with measured and published distances, but only five $\mathrm{T}$ dwarfs published to date. In this paper, we discuss two applications of the parallax data to our understanding of $L$ dwarfs.

\section{Predicting Absolute Magnitudes}

In Figure 1, the absolute magnitude $M_{J}$ is plotted. ( $M_{J}$ is used because it scales more closely with luminosity than $M_{I}$ or $M_{K}$ do.) The five known binary pairs 


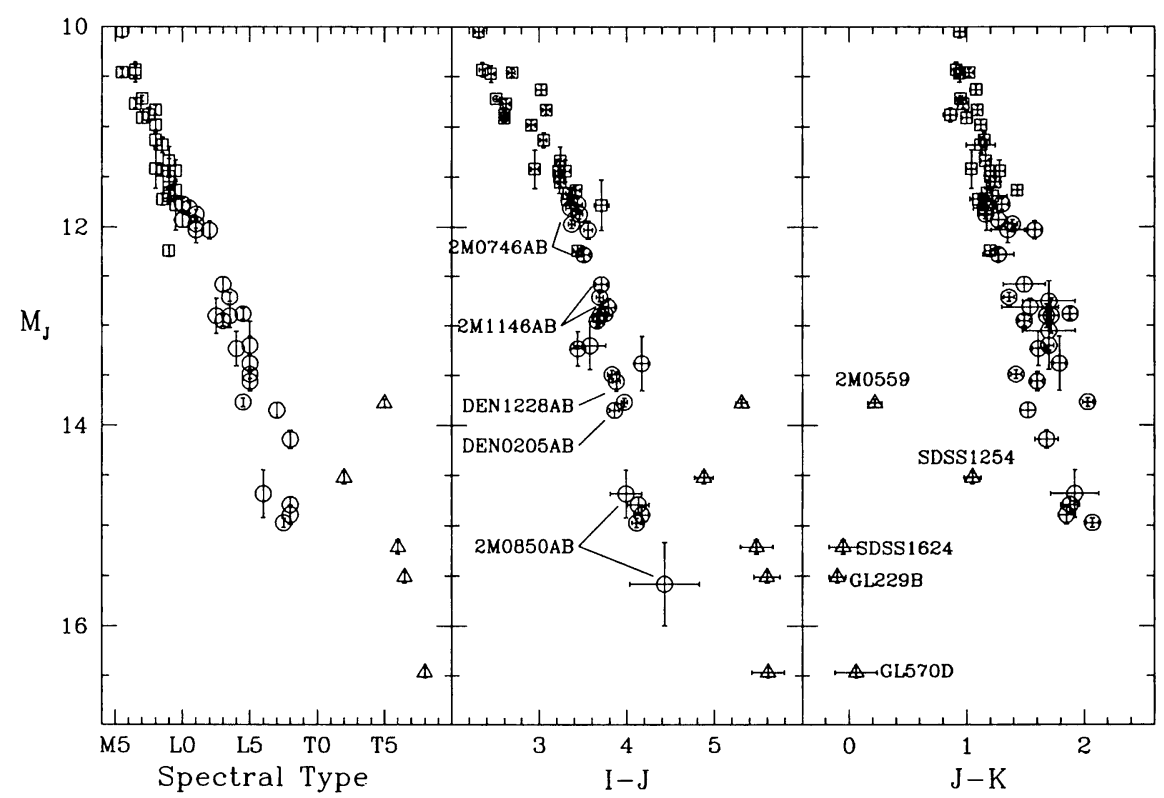

Figure 1. The absolute magnitude $M_{J}$ plotted against spectral type and color. $\mathrm{M}$ dwarfs are shown by squares, $\mathrm{L}$ dwarfs by circles, and $\mathrm{T}$ dwarfs by triangles. Binary L dwarfs are labelled in the middle panel, and $\mathrm{T}$ dwarfs are labelled in the right-hand panel.

of $\mathrm{L}$ dwarfs are labelled in the middle panel - they are plotted after correcting their magnitudes from the combined light to the individual components. The figure shows that both spectral type an-1 $I-J$ color can be used to predict $M_{J}$ for M7 through L8 spectral types. The scatter in the $J-K$ color of L dwarfs makes that color less accurate for use in predicting $M_{J}$. We need more data before drawing conclusions about $\mathrm{T}$ dwarfs.

\section{The Near-IR Colors of L Dwarfs}

The $J H K$ colors of $\mathrm{L}$ dwarfs are known to have a poor correlation with spectral type (e.g. Leggett et al. 2002) and with absolute magnitude and temperature (Kirkpatrick et al. 2000; Dahn et al. 2002). The right-hand panel of Figure 1 reinforces that conclusion. The $J H K$ colors are believed to be controlled by the amount of dust in the atmospheres of these cool objects: the distribution of dust particle sizes, the vertical distribution of dust clouds, and perhaps the existence of holes or bands between dust clouds are likely to be relevant factors (e.g. Ackerman \& Marley 2001; Burrows et al. 2002; Tsuji 2002).

In an effort to understand what factor(s) cause dust to vary between otherwise-similar objects, in Figure 2 we repeat the right-hand panel of Figure 1 to 


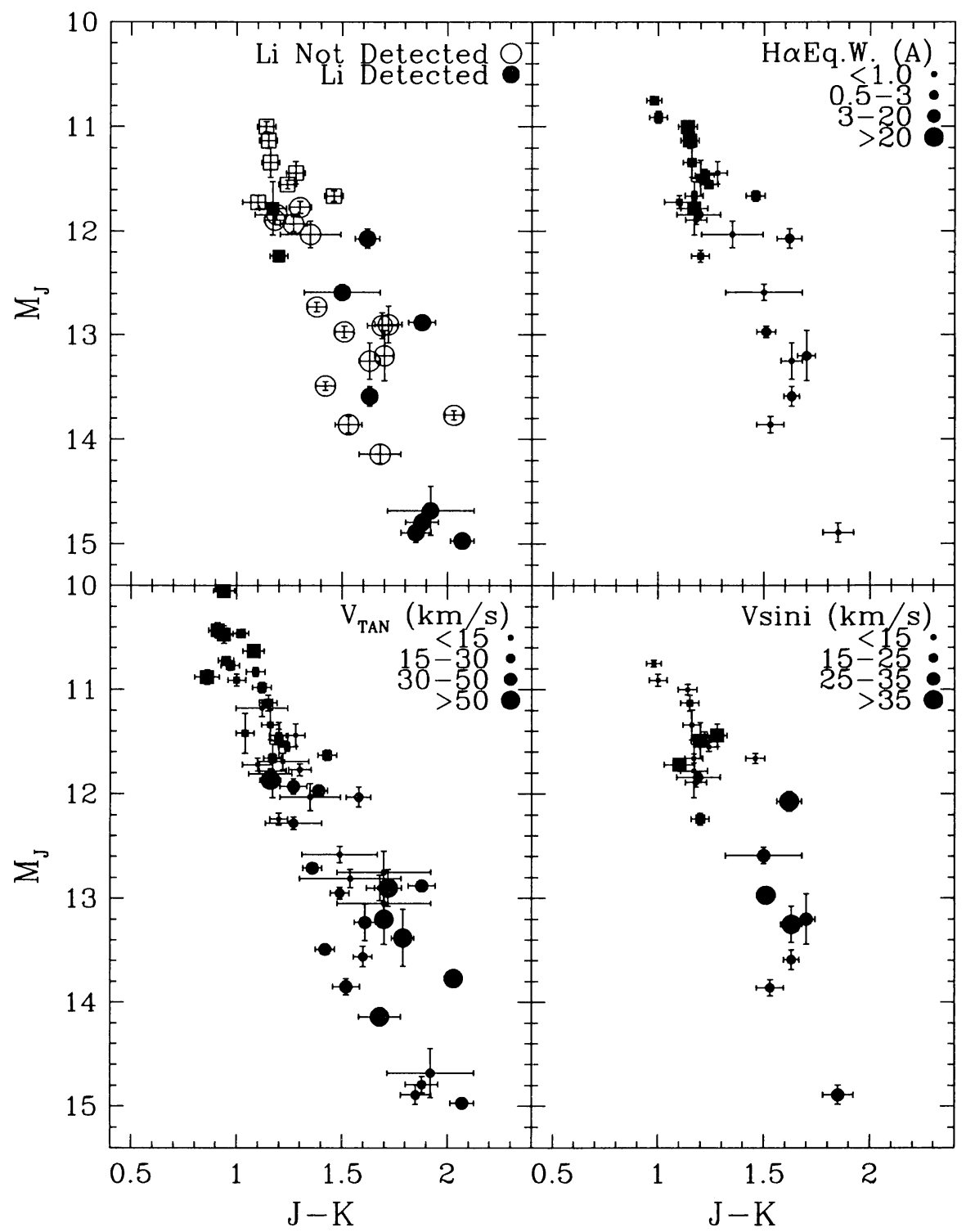

Figure 2. The near-IR color-absolute magnitude diagram for late-M and $\mathrm{L}$ dwarfs. At a given absolute magnitude, the $J-K$ color shows little correlation with any of these four parameters. 
show four potential correlations. Figure 2 uses data from the literature (Basri et al. 2000; Basri 2001; Kirkpatrick et al. 1999; 2000; 2001; Martín et al. 1999; Reid et al. 2002; Tinney \& Reid 1998) for lithium detection, $\mathrm{H} \alpha$ emission, tangential velocity, and rotation velocity. Some trends are already known: lithium is detected more frequently at fainter absolute magnitudes, while $\mathrm{H} \alpha$ equivalent widths drop and rotation velocities increase. However, based on the available data, there is no obvious tendency for the $J-K$ color to correlate with any of these parameters at a given absolute magnitude. Perhaps several factors influence the dust content of the atmospheres of $\mathrm{L}$ dwarfs and mask any one-parameter correlation.

\section{Future work}

We need data (parallax and other) for more $\mathrm{L}$ dwarfs. We really need data for more $\mathrm{T}$ dwarfs. Parallaxes for more $\mathrm{T}$ dwarfs will be coming from Tinney (this volume) and from parallaxes with the USNO IR camera (Vrba et al. in preparation).

Acknowledgments. Many objects discussed here were first identified from the 2MASS and SDSS surveys, and we are grateful to our colleagues for notifying us of objects in advance of publication.

\section{References}

Ackerman, A., \& Marley, M. 2001, ApJ, 556, 872

Basri, G. 2001, in ASP Conf. Ser. 223, 11th Cool Stars Workshop, eds. GarcíaLópez, R. Rebolo, \& M.R. Zapatero-Osorio, (San Francisco: ASP), 261

Basri, G., Mohanty, S., Allard, F., Hauschildt, P.H., Delfosse, X., Martín, E.L., Forveille, T., \& Goldman, B. 2000, ApJ, 538, 363

Burrows, A., Burgasser, A.J., Kirkpatrick, J.D., Liebert, J., Milsom, J.A., Sudarsky, M.D., \& Hubeny, I. 2002, ApJ, 573, 394

Dahn, C.C., et al. 2002, AJ, 124, 1170

Leggett, S.K., et al. 2002, ApJ, 564, 452

Kirkpatrick, J.D., et al. 1999, ApJ, 519, 802

Kirkpatrick, J.D., et al. 2000, AJ, 120, 477

Kirkpatrick, J.D., Dahn, C.C., Monet, D.G., Reid, I.N., Gizis, J.E., Liebert, J., \& Burgasser, A.J. 2001, AJ, 121, 3235

Martín, E.L., Delfosse, X., Basri, G., Goldman, B., Forveille, T., \& Zapatero Osorio, M.R. 1999, AJ, 118, 2466

Reid, I.N., Kirkpatrick, J.D., Liebert, J., Gizis, J.E., Dahn, C.C., \& Monet, D.G. 2002, AJ, 124, in press, astro-ph/0204285

Tinney, C.G. \& Reid, I.N. 1998, MNRAS, 301, 1031

Tsuji, T. 2002, ApJ, 575, 264 